\title{
Термоэлектрические свойства твердых растворов с катионным и анионным замещением на основе слоистого тетрадимитоподобного соединения $\mathrm{GeSnSb}_{4} \mathrm{Te}_{8}$
}

\author{
(C) Г.Р. Гурбанов ${ }^{1}$, Т.А. Джафраров ${ }^{2}$, М.Б. Адыгезалова ${ }^{1}$ \\ ${ }^{1}$ Азербайджанский государственный университет нефти и промышленности, \\ Az-1010 Баку, Азербайджан \\ ${ }^{2}$ Азербайджанский государственный педагогический университет, \\ Az -1000 Баку, Азербайджан \\ E-mail: ebikib@mail.ru; ceferovtapd75@gmail.com; mehpareadigozelova@yahoo.com
}

Поступила в Редакцию 18 января 2021 г.

В окончательной редакции 21 января 2021 г.

Принята к публикации 21 января 2021 г.

Термоэлектрические свойства четвертного соединения $\mathrm{GeSnSb}_{4} \mathrm{Te}_{8}$ и твердых растворов $\mathrm{GeSnSb}_{4-x} \mathrm{Bi}_{x} \mathrm{Te}_{8-y} \mathrm{Se}_{y}$ исследованы в широком интервале температур 300-600 K. Замещение атомов $\mathrm{Sb}$ атомами Вi и атомов Те атомами Se в твердых растворах приводит к росту коэффициента термоэдс и уменьшению решеточной составляющей теплопроводности по сравнению с соответствующими характеристиками $\mathrm{GeSnSb}_{4} \mathrm{Te}_{8}$. Более низкие значения решеточной теплопроводности в сплавах с $x=0.6, y=0.4$ по сравнению $\mathrm{c} \mathrm{GeSnSb}_{4} \mathrm{Te}_{8}$ связаны с искажениями из-за различия атомных масс и размеров атомов $\mathrm{Sb}$ и $\mathrm{Bi}$, а также Те и $\mathrm{Se}$. Показано, что с увеличением количества атомов, участвующих в замещениях в обеих подрешетках при образовании твердого раствора, максимум температурной зависимости термоэдс и минимум температурной зависимости теплопроводности сдвигаются в область более высоких температур, что обусловлено увеличением ширины запрещенной зоны. При увеличении содержания Вi и $\mathrm{Se}$ в твердых растворах уменьшается решеточная теплопроводность и соответственно увеличивается термоэлектрическая эффективность. Термоэлектрическая эффективность образца $\mathrm{GeSnSb}_{4-x} \mathrm{Bi}_{x} \mathrm{Te}_{8-y} \mathrm{Se}_{y}$ $(x=0.6, y=0.4)$ имеет максимальное значение $Z=3.34 \cdot 10^{-3} \mathrm{~K}^{-1}$ при $300 \mathrm{~K}$.

Ключевые слова: $\mathrm{GeSnSb}_{4} \mathrm{Te}_{8}$, твердые растворы $\mathrm{GeSnSb}_{4-x} \mathrm{Bi}_{x} \mathrm{Te}_{8-y} \mathrm{Se}_{y}$, коэффициент термоэдс, теплопроводность, электропроводность, термоэлектрическая эффективность.

DOI: 10.21883/FTP.2021.05.50826.9610

\section{1. Введение}

Прогресс современной техники и современных технологий тесно связан с поиском новых источников энергии, наиболее универсальных для практического использования. Здесь на первый план выходят требования экологической чистоты ее выработки и возобновляемости источников. С этой точки зрения термоэлектричество относится к перспективным способам прямого преобразования тепловой энергии в электрическую без промежуточного этапа перехода в механическую энергию, как это происходит на тепловых и атомных электростанциях [1-4].

Получение и преобразование энергии является одним из важнейших направлений деятельности современной цивилизации. Большое внимание было обращено в этой связи на твердотельные термоэлектрические преобразователи, которые имеют ряд преимуществ перед традиционными электрическими генераторами и холодильниками: простота конструкции, отсутствие движущихся частей, бесшумность работы, высокая надежность, возможность миниатюризации без потери эффективности. Однако для масштабных промышленных применений термоэлектрических преобразователей энергии необходимо существенное повышение их эффективности, кото- рое в первую очередь связано с использованием более эффективных термоэлектрических материалов.

Термоэлектричество представляет собой совокупность физических явлений (эффекты Зеебека, Пельтье, Томпсона), в которых при наличии градиента температур возникает разность потенциалов. На основе данных эффектов разработаны системы термоэлектрического преобразования энергии: термоэлектрические генераторы (ТЭГ), преобразующие тепловую энергию в электрическую, и термоэлектрические охладители. Достоинство таких преобразователей состоит в надежности, отсутствии движущихся частей, возможности использования в условиях, в которых невозможно использовать традиционные источники энергии (космос, районы Крайнего Севера) и др. [5-7]. Основной проблемой в данной области является низкая эффективность преобразования.

Эффективность термоэлектрического генератора $(\eta)$, а именно эффективность преобразования тепловой энергии в электрическую, определяется формулой

$$
\eta=\frac{\Delta T}{T_{h}} \frac{\sqrt{1+Z T}-1}{\sqrt{1+Z T}+T_{c} / T_{h}},
$$

где $T_{h}$ - температура на горячей стороне термоэлемента, $T_{c}$ - температура на холодной стороне термоэлемента, $\Delta T-$ разность температур между горячим 
и холодным концами, $Z T$ - безразмерный параметр добротности термоэлектрического материала.

В современных ТЭГ эффективность преобразования не превышает 8\% [8-10]. Для повышения эффективности преобразования ведется поиск новых термоэлектрических материалов с высоким значением параметра добротности $Z T$, который описывается формулой

$$
Z T=\frac{\sigma S^{2}}{\kappa T}
$$

где $\sigma$ - электропроводность, $S-$ коэффициент Зеебека, $\kappa$ - теплопроводность, $T-$ температура [11-14].

При этом стоит учитывать, что каждый материал имеет максимум термоэлектрической добротности в определенной области температур, что определяет область его применения [15-17].

В отличие от других альтернативных способов преобразования энергии, термоэлектричество не зависит от погодных условий. Для его реализации необходимо иметь постоянный источник тепла, такой как: отходящее тепло выхлопных газов двигателя, отходящее тепло процессора, тепло человека и др. Также современный прогресс в миниатюризации устройств предполагает возможность применения термоэлектрических генераторов для энергетической независимости таких устройств $[18,19]$. Наряду с эффективностью преобразования существует ряд других требований, которые определяют возможность использования термоэлектричества, - например, нетоксичность используемых материалов, способ получения материала и многое другое. Таким образом, поиск новых термоэлектрических материалов является актуальной областью исследований.

Полупроводниковые материалы по праву занимают одно из ведущих мест в ряду важнейших материалов, определяющих уровень развития мировой цивилизация. Они составляют основу элементной базы современной электронной техники, без которой сегодня немыслим научно-технический прогресс. С развитием твердотельной электроники (прежде всего микроэлектроники) связано успешное решение проблем крупномасштабной компьютеризации и информатизации, создания современных систем связи, телевидения, эффективной передачи и преобразования электроэнергии, разнообразной бытовой, медицинской и специальной электронной аппаратуры. Большую роль играют эти материалы в решении задач развития экологически чистых энергетики и холодильной техники, создания современных систем мониторинга загрязнений окружающей среды, а также высокочувствительной сенсорной техники широкого функционального назначения [20].

Для создания современных полупроводниковых приборов все большее применение находят многокомпонентные твердые растворы, позволяющие в широких переделах варьировать параметры материала. Однако известно, что при температурах ниже линии солидуса возможен нежелательный распад твердых растворов, приводящий к изменению эксплуатационных характеристик материала, в связи с чем актуальна оценка стабильности применяемых твердых растворов [21].

Полупроводниковые твердые растворы в системах со значительной растворимостью в твердой фазе традиционно являются предметом интенсивных исследований, поскольку для них характерно монотонное изменение многих свойств (например, ширины запрещенной зоны, параметра элементарной ячейки), что позволяет варьировать функциональные характеристики материала [22].

Полупроводниковые вещества $\mathrm{A}^{\mathrm{IV}} \mathrm{B}^{\mathrm{VI}}$ являются перспективными материалами в различных областях электроники: детекторы и источники инфракрасного излучения, термоэлектрические элементы, солнечные батареи, элементы памяти, спинтроники и т.д. Фундаментальные характеристики этих соединений - малая ширина запрещенной зоны, высокая диэлектрическая проницаемость, радиационная стойкость, высокие значения подвижности носителей зарядов, высокая ионность связи и ряд других - являются уникальными среди полупроводниковых соединений и твердых растворов [23].

Одним из быстро развивающихся в настоящее время направлений поиска является создание тройных или четверных узкозонных полупроводников со сложными кристаллическими решетками [24-26].

Халькогениды типа $\mathrm{A}^{\mathrm{V}} \mathrm{Sb}^{\mathrm{VI}}(\mathrm{A}=\mathrm{Sb}, \mathrm{Bi} ; \mathrm{B}=\mathrm{S}, \mathrm{Se}, \mathrm{Te})$ привлекают разработчиков приборов благодаря своим термоэлектрическим и фотоэлектрическим свойствам. В то же время низкая температура плавления и низкая механическая прочность этих материалов ограничивают возможности их применения [27,28].

Халькогениды, в частности теллуриды и селениды, представляют интерес также для создания топологических изоляторов [29-32].

Таким образом, как следует из изложенного, необходимо дальнейшее изучение химического взаимодействия между халькогенидными соединениями, чтобы расширить круг полупроводниковых материалов и получать составы с заданными физическими свойствами.

Полупроводниковые твердые растворы в системах со значительной растворимостью в твердой фазе традиционно являются предметом интенсивных исследований, поскольку для них характерно монотонное изменение многих параметров (например, ширины запрещенной зоны, параметров элементарной ячейки), что позволяет варьировать функциональные свойства материала, меняя состав.

В тройных и четверных смешанных слоистых халькогенидах можно достичь существенно более низких значений решеточной теплопроводности по сравнению с исходными компонентами, что делает их перспективными термоэлектрическими материалами $[4,33]$.

C этой точки зрения смешанные кристаллы $\mathrm{GeSnSb}_{4} \mathrm{Te}_{8}$, легированные $\mathrm{Bi}$, Se и $\mathrm{S}$, представляют особый интерес. В системе $\mathrm{GeSb}_{2} \mathrm{Te}_{4} \mathrm{SnSb}_{2} \mathrm{Te}_{4}$ при соотношении $\mathrm{GeSb}_{2} \mathrm{Te}_{4}: \mathrm{SnSb}_{2} \mathrm{Te}_{4}=1: 1 \quad$ образуется четверное соединение. Соединение $\mathrm{GeSnSb}_{4} \mathrm{Te}_{8}$ крис- 
таллизуется в ромбической сингонии с параметрами $a=4.92 \AA, b=9.70 \AA, c=11.28 \AA$; пр. гр. Рnnm, объем элементарной ячейки $V=837.44 \AA^{3}$; заряд $z=2[34]$.

Цель работы заключалась в исследовании влияния замещений в катионной и анионной подрешетках $\mathrm{GeSnSb}_{4} \mathrm{Te}_{8}$ на термоэлектрические свойства.

\section{2. Методика эксперимента}

Для исследования термоэлектрических свойств были приготовлены твердые растворы составов $\mathrm{GeSnSb}_{4} \mathrm{Te}_{8}$ и $\mathrm{GeSnSb}_{4-x} \mathrm{Bi}_{x} \mathrm{Te}_{8-y} \mathrm{Se}_{y}$. В качестве исходных материалов использовались германий с удельным сопротивлением 10 Ом·см, олово марки ОВЧ-000, висмут, сурьма и теллур с чистотой $99.999 \%$, а также селен и сера марки ВЧ. Легирование материалов осуществлялось с помощью хлористого кадмия, эффективный коэффициент распределения которого был близок к 1. Для получения образцов использовался один из методов направленной кристаллизации - вертикальное зонное выравнивание. Скорость кристаллизации и градиент температуры на фронте кристаллизации обеспечивали условия, при которых концентрационного переохлаждения не возникало. Этот метод позволял получать образцы, состоящие из нескольких монокристаллических блоков, плоскости спайности которых совпадали с направлением роста кристалла. Полученные в результате кристаллизации слитки длиной $110-130$ мм и диаметром 7-9 мм разрезались с помощью электроискровой резки на образцы длиной 10-15 мм. Измерения электропроводности $\sigma$, теплопроводности $\kappa$ и коэффициента термоэдс $\alpha$ осуществлялись вдоль плоскостей спайности, по которым были направлены электрический ток и тепловой поток. Все измерения указанных параметров проводились на постоянном токе.

Для проведения исследования термоэлектрических свойств соединения $\mathrm{GeSnSb}_{4} \mathrm{Te}_{8}$ и установления влияния замещений в катионной и анионной подрешетках на свойства соединения была синтезирована следующая серия образцов: $\mathrm{GeSnSb}_{4} \mathrm{Te}_{8}$ и $\mathrm{GeSnSb}_{4-x} \mathrm{Bi}_{x} \mathrm{Te}_{8-y} \mathrm{Se}_{y}$ c $(x=0.2, \quad y=0.18), \quad(x=0.4, \quad y=0.3), \quad(x=0.6$, $y=0.4)$.

\section{3. Результаты эксперимента и обсуждение}

Температурные зависимости электропроводности, коэффициента термоэдс, теплопроводности $\mathrm{GeSnSb}_{4} \mathrm{Te}_{8}$ и твердых растворов $\mathrm{GeSnSb}_{4-x} \mathrm{Bi}_{x} \mathrm{Te}_{8-y} \mathrm{Se}_{y}$ измеряли в интервале температур $T=300-600 \mathrm{~K}$. В таблице представлены результаты измерения термоэлектрических характеристик при $300 \mathrm{~K}$. Все сплавы относятся к классу указанных полупроводников с электронным типом проводимости. Как видно из таблицы, сплавы характеризуются очень низкими значениями решеточной составляющей теплопроводности и с увеличением

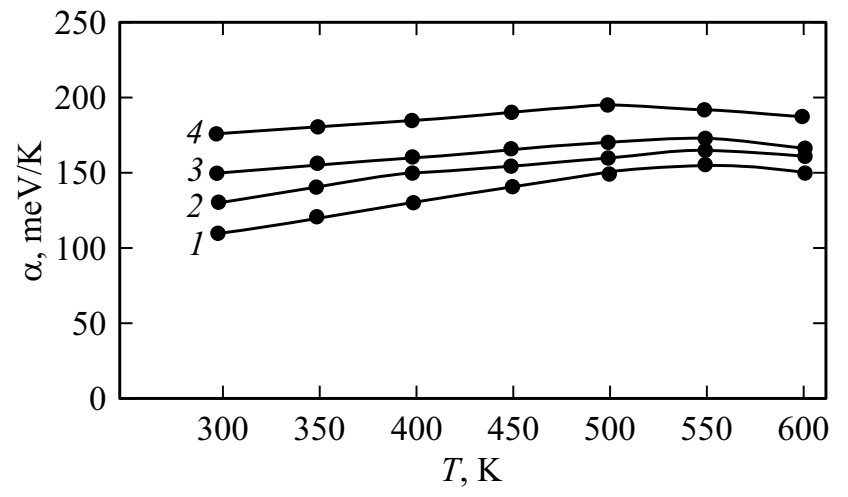

Рис. 1. Температурная зависимость коэффициента термоэдс для сплавов $\mathrm{GeSnSb}_{4} \mathrm{Te}_{8}$ и $\mathrm{GeSnSb}_{4-x} \mathrm{Bi}_{x} \mathrm{Te}_{8-y} \mathrm{Se}_{y} .2-$ $(x=0.2, y=0.18), 3-(x=0.4, y=0.3), 4-(x=0.6$, $y=0.48)$.

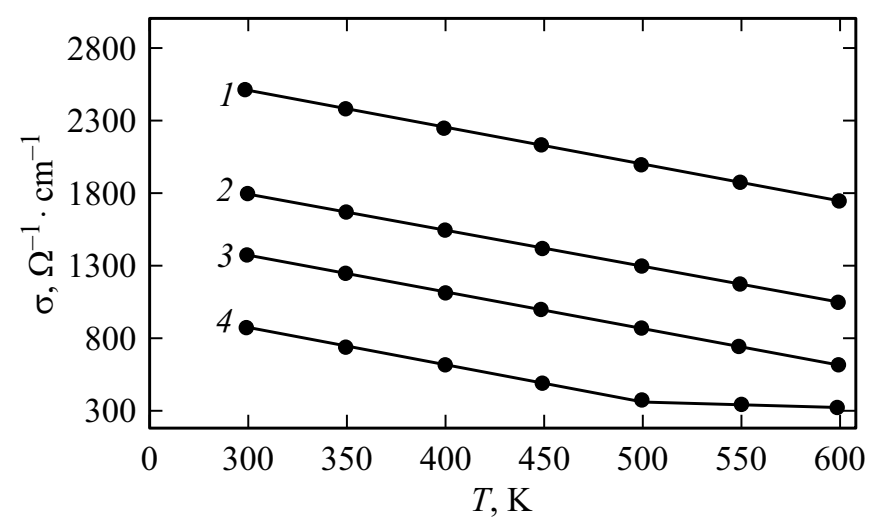

Рис. 2. Температурные зависимости электропроводности для сплавов $\mathrm{GeSnSb}_{4} \mathrm{Te}_{8}$ и $\mathrm{GeSnSb}_{4-x} \mathrm{Bi}_{x} \mathrm{Te}_{8-y} \mathrm{Se}_{y}$. (1-4) - то же, что на рис. 1 .

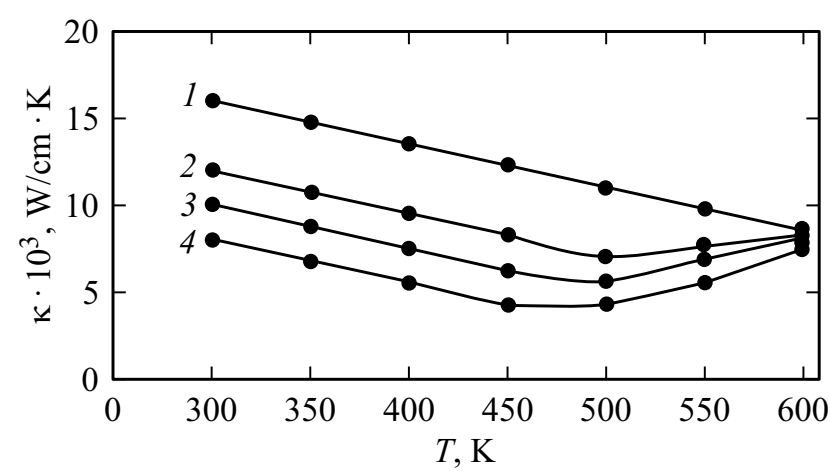

Рис. 3. Температурные зависимости теплопроводности для сплавов $\mathrm{GeSnSb}_{4} \mathrm{Te}_{8}$ и $\mathrm{GeSnSb}_{4-x} \mathrm{Bi}_{x} \mathrm{Te}_{8-y} \mathrm{Se}_{y}$. (1-4) - то же, что на рис. 1,2 .

количества атомов, участвующих в замещениях в обеих подрешетках при образовании твердого раствора, теплопроводность уменьшается.

На рис. 1-3 представлены температурные зависимости коэффициента термоэдс, электропроводности и 
Кинетических параметров сплавов $\mathrm{GeSnSb}_{4} \mathrm{Te}_{8}$ и $\mathrm{GeSnSb}_{4-x} \mathrm{Bi}_{x} \mathrm{Te}_{8-y} \mathrm{Se}_{y}$ при температуре $T=300 \mathrm{~K}$

\begin{tabular}{c|c|c|c|c}
\hline Образец & $\begin{array}{c}\alpha, \\
\mathrm{MKB} / \mathrm{K}\end{array}$ & $\begin{array}{c}\sigma, \\
\mathrm{OM}^{-1} \cdot \mathrm{cm}^{-1}\end{array}$ & $\begin{array}{c}\kappa, \\
10^{-3} \mathrm{BT} / \mathrm{cm} \cdot \mathrm{K}\end{array}$ & $\begin{array}{c}Z, \\
\mathrm{~K}^{-1}\end{array}$ \\
\hline $\mathrm{GeSnSb}_{4} \mathrm{Te}_{8}$ & -110 & 2500 & 16 & $1.90 \cdot 10^{-3}$ \\
$\mathrm{GeSnSb}_{4-x} \mathrm{Bi}_{x} \mathrm{Te}_{8-y} \mathrm{Se}_{y} x=0.2, y=0.18$ & -130 & 1800 & 12 & $2.53 \cdot 10^{-3}$ \\
$\mathrm{GeSnSb}_{4-x} \mathrm{Bi}_{x} \mathrm{Te}_{8-y} \mathrm{Se}_{y} x=0.4, y=0.3$ & -150 & 1375 & 10 & $3.09 \cdot 10^{-3}$ \\
$\mathrm{GeSnSb}_{4-x} \mathrm{Bi}_{x} \mathrm{Te}_{8-y} \mathrm{Se}_{y} x=0.6, y=0.4$ & -175 & 875 & 8 & $3.34 \cdot 10^{-3}$
\end{tabular}

теплопроводности в широком интервале температур $(300-600 \mathrm{~K})$. Исследованные сплавы относятся к категории сильно легированных полупроводников и характеризуются высокой степенью разупорядочения.

Как видно из рис. 1 и 2, коэффициент термоэдс возрастает, а электропроводность падает с температурой для всех четырех составов.

Величина термоэдс в $\mathrm{GeSnSb}_{4} \mathrm{Te}_{8}$ и в твердых растворах халькогенидов висмута и сурьмы с ростом температуры возрастает в области примесной проводимости, достигает максимума при разных температурах, зависящих от состава раствора и концентрации носителей, и затем с наступлением смешанной проводимости уменьшается. Сравнение температурных зависимостей термоэдс образцов разных составов показывает, что температура максимума возрастает с увеличением количества атомов, участвующих в замещении при образовании твердого раствора. По знаку термоэдс ясно, что все четыре образца имеют проводимость $n$-типа. Исследованные сплавы сохраняют $n$-тип проводимости во всем изученном интервале температур. Максимум коэффициента термоэдс находится при $500 \mathrm{~K}$.

На рис. 2 приведены температурные зависимости электропроводности, из которых видно, что для всех четырех составов удельная электропроводность образцов с уменьшением температуры растет и температурная зависимость электропроводности носит металлический характер. Такой вид температурной зависимости электропроводности характерен для частично вырожденных полупроводников, где участвуют два механизма рассеяния носителей заряда: на заряженных примесях и тепловых колебаниях решетки.

Температурные зависимости полной теплопроводности образцов $\mathrm{GeSnSb}_{4-x} \mathrm{Bi}_{x} \mathrm{Te}_{8-y} \mathrm{Se}_{y}$ и $\mathrm{GeSnSb}_{4} \mathrm{Te}_{8}$ приведены на рис. 3 , из которого видно, что характер изменения к в указанном интервале температур существенно отличается от зависимости в примесной области, где $\kappa$ уменьшается с ростом температуры. Это уменьшение $\kappa$ обусловлено в основном увеличением рассеяния на тепловых колебаниях решетки. Замещение атомов $\mathrm{Sb}$ атомами $\mathrm{Bi}$ и атомов Те атомами Se приводит к росту коэффициента термоэдс и уменьшению решеточной составляющей теплопроводности по сравнению с соответствующими свойствами $\mathrm{GeSnSb}_{4} \mathrm{Te}_{8}$. Более низкие значения решеточной теплопроводности в сплавах с $x=0.6, y=0.4$ по сравнению $\mathrm{c} \mathrm{GeSnSb}_{4} \mathrm{Te}_{8}$ связаны с искажениями из-за различия атомных масс и размеров атомов $\mathrm{Sb}$ и $\mathrm{Bi}$, а также Те и $\mathrm{Se}$. Показано, что с увеличением количества атомов, участвующих в замещениях в обеих подрешетках при образовании твердого раствора, максимум температурной зависимости термоэдс и минимум температурной зависимости теплопроводности сдвигаются в область более высоких температур, что обусловлено увеличением ширины запрещенной зоны.

Таким образом, сопоставляя характер температурных зависимостей электропроводности, теплопроводности и коэффициента термоэдс в области смешанной проводимости в $\mathrm{GeSnSb}_{4} \mathrm{Te}_{8}$ и твердых растворах $\mathrm{GeSnSb}_{4-x} \mathrm{Bi}_{x} \mathrm{Te}_{8-y} \mathrm{Se}_{y}$, можно сделать вывод о том, что появление неосновных носителей приводит к уменьшению термоэдс и к росту полной теплопроводности, при этом существенного увеличения электропроводности не наблюдается.

Анализируя в целом результаты исследования термоэлектрических свойств тетрадимитоподобного соединения $\mathrm{GeSnSb}_{4} \mathrm{Te}_{8}$ и твердого раствора $\mathrm{GeSnSb}_{4-x} \mathrm{Bi}_{x} \mathrm{Te}_{8-y} \mathrm{Se}_{y}$, следует отметить, что эти образцы являются перспективными для создания термоэлектрических материалов с низкой решеточной теплопроводностью.

\section{4. Заключение}

Таким образом, впервые исследованы температурные зависимости основных термоэлектрических параметров тетрадимитоподобного соединения $\mathrm{GeSnSb}_{4} \mathrm{Te}_{8}$ и твердого раствора $\mathrm{GeSnSb}_{4-x} \mathrm{Bi}_{x} \mathrm{Te}_{8-y} \mathrm{Se}_{y}$.

Установлено, что замещение атомов $\mathrm{Sb}$ атомами Вi и атомов Те атомами Se приводит к росту коэффициента термоэдс и уменьшению решеточной составляющей теплопроводности по сравнению с соответствующими величинами для $\mathrm{GeSnSb}_{4} \mathrm{Te}_{8}$.

Двойные замещения в катионной и анионной подрешетках увеличивают термоэлектрическую эффективность относительно $\mathrm{GeSnSb}_{4} \mathrm{Te}_{8}$. Максимальное значение получено для образца $\mathrm{GeSnSb}_{4-x} \mathrm{Bi}_{x} \mathrm{Te}_{8-y} \mathrm{Se}_{y}$ $(x=0.6, y=0.4)$, оно составляет $Z=3.34 \cdot 10^{-3} \mathrm{~K}^{-1}$ при $300 \mathrm{~K}$.

\section{Конфликт интересов}

Авторы заявляют, что у них нет конфликта интересов. 


\section{Список литературы}

[1] M.G. Kanatzidis. Acc. Chem. Res., 38 (4), 361 (2005). https://doi.org/10.1021/ar040176w

[2] Л.Е. Шелимова, О.Г. Карпинский, В.С. Земсков. Перспективные материалы, № 5, 23 (2000).

[3] А.В. Шевелькова. Успехи химии, 77 (1), 3 (2008). https://doi.org/10.1070/RC2008v077n01ABEH003746

[4] M.G. Kanatzidis. Semiconductors and Semimetals, ed. by T.M. Tritt (San Diego-San Francisco-N.Y.-Boston-LondonSydney-Tokyo, Academic Press, 2001) v. 69, p. 51. https://doi.org/10.1016/S0080-8784(01)80149-6

[5] А.Ф. Иоффе. Полупроводниковые термоэлементы (М., AH CCCP, 1960).

[6] X. Zhang, L.-D. Zhao. J. Materiomics, 1, 92 (2015). https://doi.org/10.1016/j.jmat.2015.01.001

[7] J.C. Zheng. Front. Phys. China, 3 (5), 269 (2008).

[8] S. Twaha, J. Zhu, Y. Yan, B. Li. Renewable Sustainable Energy Rev., 65, 698 (2016). DOI: $10.1016 /$ j.rser.2016.07.034

[9] A. Sharma, J.H. Lee, K.H. Kim, J.P. Jung. J. Microelectron. Packag. Soc., 24 (1), 9 (2017). https://doi.org/10.6117/kmeps.2017.24.1.009

[10] S. Patidar. Int. J. Res. Appl. Sci. Eng. Technol., 6 (5), 1992 (2018). DOI: 10.22214/ijraset.2018.5325

[11] D. Champier. Energy Convers. Manage., 140, 167 (2017). DOI: 10.1016/j.enconman.2017.02.070

[12] I. Petsagkourakis, K. Tybrandt, X. Crispin, I. Ohkubo, N. Satoh, T. Mori. Sci. Technol. Adv. Mater., 19 (1), 836 (2018). DOI: $10.1080 / 14686996.2018 .1530938$

[13] E. Kanimba, M. Pearson, J. Sharp, D. Stokes, S. Priya, Z. Tian. Energy, 142, 813 (2018). https://doi.org/10.1016/j.energy.2017.10.067

[14] K. Gaurav, S.K. Pandey. J. Renewable Sustainable Energy, 9 (1), 014701-1-014701-13 (2017). https://doi.org/10.1063/1.4976125

[15] H.S. Kim, K. Kikuchi, T. Itoh, T. Iida, M. Taya. Mater. Sci. Eng. B, 185, 45 (2014). https://doi.org/10.1016/j.mseb.2014.02.005

[16] K. Huang, Y. Yan, B. Li, Y. Li, K. Li, J. Li. Automotive Innovation, 1, 54 (2018). https://doi.org/10.1007/s42154-018-0006-Z

[17] W. Liu, J. Hu, S. Zhang, M. Deng, C.-G. Han, Y. Liu. Materials Today Physics, 1, 50 (2017). https://doi.org/10.1016/j.mtphys.2017.06.001

[18] J. Carmo, L. Goncalves, R. Wolffenbuttel, J. Correia. Sensors Actuators A, 161 (1-2), 199 (2010). 10.1016/j.sna.2010.05.010

[19] B. Ramachandran, K.K. Wu, Y.K. Kuo, M.S. Ramachandra Rao. J. Phys. D: Appl. Phys., 48 (11), 115301 (2015).

[20] М.Г. Мильвидский, В.Б. Уфимцев. Неорг. матер., 36 (3), 360 (2000).

[21] Т.П. Сушкова, Г.В. Семенова, Е.В. Стрычина. Вестн. ВГУ, 1, 94 (2004).

[22] А.А. Волыхов, Л.В. Яшина, В.И. Штанов. Неорг. матер., 72 (6), 662 (2006).

[23] А.А. Волыхов, Л.В. Яшина, М.Е. Тамм, А.В. Рыженков. Неорг. матер., 45 (9), 1042 (2009).

[24] A.R. West. Solid State Chemistry and its Applications, 2nd edn (N.Y., Wiley, 2014).

[25] Л.Д.Иванова, Л.И. Петрова, Ю.В. Гранаткина. Неорг. матер., 52 (3), 289 (2016).

https://doi.org/10.7868/S0002337X16030040
[26] С.А. Асадов, А.Н. Мамедов, С.А. Кулиева. Неорг. матер.. 52 (9), 942 (2016). DOI: https://doi.org/10.7868/S0002337X16090013

[27] Л.И. Анатыкуч. Термоэлементы и термоэлектрические устройства (Киев, Наук. думка, 1940).

[28] L.A. Kuznetsov, D.A. Rowe. J. Appl. Phys., 34 (5), 700 (2001).

[29] M. Caputo, M. Panighel, S. Lisi, L. Khalil, G. Di Santo, E. Papalazarou, A. Hruban, M. Konczykowsky, L. KrusinElbaum, Z.S. Aliev. Nano Lett., 16, 3409 (2016). https://doi.org/10.1021/acs.nanolett.5b02635

[30] M. Papagno, S.V. Eremeev, J. Fujii, Z.S. Aliev, M.B. Babanly, S.K. Mahatha, I. Vobornik, N.T. Mamedov, D. Pacile, E.V. Chulkov. ACS Nano, 10, 3518 (2016). https://doi.org/10.1021/acsnano.5b07750

[31] C. Lamuta, D. Campi, A. Cupolillo, Z. Aliev, M. Babanly, E. Chulkov, A. Politano, L. Pagnotta. Scripta Mater., 121, 50 (2016). DOI: 10.1016/j.scriptamat.2016.04.036

[32] L. Viti, D. Coquillat, A. Politano, K.A. Kokh et. al. Nano Lett., 16 (7), 80 (2016). https://doi.org/10.1021/acs.nanolett.5b02901

[33] О.Г. Карпинский, Л.Е. Шелимова, М.А. Кретова, Е.С. Авилов, В.С. Земсков. Неорг. матер., 39 (3), 305 (2003).

[34] Г.Р. Гурбанов, М.Б. Адыгезалова, А.Н. Мамедов, С.А. Гулиева. Moscow University Chem. Bull., 74 (3), 134 (2019). DOI: $10.3103 /$ S0027131419030064

Редактор Л.В. Шаронова

\section{Thermoelectric properties of solid solutions with cationic and anionic substitution on the basis of layered tetradimite-like compound $\mathrm{GeSnSb}_{4} \mathrm{Te}_{8}$}

G.R. Gurbanov' ${ }^{1}$, T.A. Jafarov' ${ }^{2}$, M.B. Adygezalova ${ }^{1}$

${ }^{1}$ Azerbaijan State University of Oil and Industry, Az-1010 Baku, Azerbaijan

${ }^{2}$ Azerbaijan State Pedagogical University, Az-1000 Baku, Azerbaijan

Abstract The thermoelectric characteristics of the $\mathrm{GeSnSb}_{4} \mathrm{Te}_{8}$ quaternary compound and $\mathrm{GeSnSb}_{4-x} \mathrm{Bi}_{x} \mathrm{Te}_{8-y} \mathrm{Se}_{y}$ solid solutions were measured in a wide temperature range $(300-600 \mathrm{~K})$. The replacement of $\mathrm{Sb}$ atoms with $\mathrm{Bi}$ atoms and $\mathrm{Te}$ atoms with $\mathrm{Se}$ atoms leads to an increase in the thermo-emf coefficient and a decrease in the lattice component of thermal conductivity in comparison with the corresponding values for $\mathrm{GeSnSb}_{4} \mathrm{Te}_{8}$. The lower values of the lattice thermal conductivity in the alloys with $x=0.6, y=0.4$ in comparison with $\mathrm{GeSnSb}_{4} \mathrm{Te}_{8}$ are associated with distortions due to the difference in the atomic masses and sizes of $\mathrm{Sb}$ and $\mathrm{Bi}$, also Te and Se atoms. It is shown that with an increase in the number of atoms participating in substitutions in both sublattices during the formation of a solid solution, the maximum of the temperature dependence of the thermo-emf and the minimum of the temperature dependence of thermal conductivity are shifted higher temperatures, which is due to an increase in the band gap. With an increase in the content of $\mathrm{Bi}$ and $\mathrm{Se}$ in solid solutions, the lattice thermal conductivity decreases and, accordingly, the thermoelectric efficiency increases. Thermoelectric figure of merit of the $\mathrm{GeSnSb}_{4-x} \mathrm{Bi}_{x} \mathrm{Te}_{8-y} \mathrm{Se}_{y}$ sample $(x=0.6, y=0.4)$ has a maximum value with $Z=3.34 \cdot 10^{-3} \mathrm{~K}^{-1}$ at $300 \mathrm{~K}$. 